\title{
Dynamic Path Restoration for New Call Blocking Versus Handoff Call Blocking In Hetrogeneous Network Using Buffers for QoS
}

\author{
A K Daniel \\ Computer Sc \& Engg Department \\ M M M Engineering College \\ GORAKHPUR (U P) India \\ danielak@rediffmail.com
}

\author{
R Singh \\ Department of CS \& I T \\ M J P Rohilkhand University \\ BAREILLY (U P) India \\ rsiet2002@gmail.com
}

\author{
J P Saini \\ Principal \\ M M M Engineering College \\ GORAKHPUR (U P) India \\ Jps_uptu@rediffmail.com
}

\begin{abstract}
An Ad hoc network is a collection of wireless mobile nodes dynamically forming a temporary network without the use of any existing heterogeneous network infrastructure or centralized administration. Routing protocols used inside ad hoc networks must be prepared to automatically adjust to an environment that can vary between the extremes of high mobility with low band width, and low mobility with high bandwidth The tremendous growth of wireless networks demands the need to meet different multimedia (such as voice audio, video, data, etc) applications available over the network. This application demand and allocation could lead to congestion if the network has to maintain such high resources for the quality of service (QoS) requirements of the applications. In this paper, a new Protocol is proposed for wireless mobile heterogeneous networks are based on the use of. path information, traffic and bandwidth resource information at each node, for allocation of route path and Handoff problem. The proposed protocol uses two buffers one for new call and another buffer is use for handoff calls if there is no channel available instead of dropping (rejecting) them it store in the buffer and when ever the channel is free it allocate for communication The protocol improved the performance of the network especially by the effect of the dynamic threshold of buffer size of new call buffer and handoff call buffer In the link failure situation we provide another path for the communication by applying a Restoration Mechanism for the survivability of link and improved the QoS of mobile network .
\end{abstract}

Keywords: Handoff call, New call buffer, Congestion, Heterogeneous network, Quality of Service (QoS),

\section{INTRODUCTION}

An ad hoc network is a collection of wireless mobile nodes dynamically forming a temporary network without the use of any existing network infrastructure or centralized administration. Ad-hoc networks are self-configuring and selfmaintaining networks that allow dispensing of fixed infrastructures. The network relies on nodes cooperation for providing packet routing. Ad-hoc network technology presents a great potential in application domains where infrastructure deployment is expensive or not possible, like battlefield environments transportation or ambient intelligence scenarios [1][2][3]. Cornerstones of ad hoc networks are routing protocols. These protocols are specifically designed to promote dissemination of routing information among network nodes. The goal is to allow the creation of communication links between any two network nodes and responsible for enabling network communications. While exchanging information, the nodes may continue to move from one place to another, so the network must be prepared to adapt continually [4][5][6]. The network infrastructure component such as repeaters, base-stations will frequently be either undesirable or not directly reachable, the nodes must be prepared to organize themselves into a network and establish routes among themselves without any outside support. In the simplest cases, the nodes may be able to communicate directly with each other.[7][8]. Future wireless networks will provide ubiquitous communication services to a large number of mobile users [9][10][11][12]. The design of such networks is based on a cellular architecture that allows efficient use of the limited available spectrum[13][14][15].

Cellular technology is designed to provide communication between two moving units, or between one mobile unit to one stationary unit. A service provider must be able to locate and track a caller, assign a channel to the caller, and transfer the channel to the call, and transfer channel from one base station to another base station as the caller moves out of cell range. Each base station is controlled by a mobile switching centre .The mobile switching centre coordinate communication between all the base station and the central office. The central office is a computerized centre that is responsible for connecting calls, recording calls information, and billing etc.

The cellular architecture consists of a backbone network with fixed base stations interconnected through a fixed network, and of mobile units that communicate with the base stations via wireless links. The geographic area within which mobile units can communicate with a particular base station is referred to a cell. Neighboring cells overlap with each other, thus ensuring continuity of communications when the users move from one cell to another. The mobile units communicate with each other, as well as with other networks, through the base stations and backbone network. A set of channels (frequencies) is allocated to each base station. Neighboring 
cells have to use different channels in order to avoid intolerable interferences (we do not consider CDMA networks). Many dynamic channel allocation algorithms have been proposed [16][17][18]. These algorithms may improve the performances of the cellular networks. However the channel allocation is usually done in a static way. When a mobile user wants to communicate with another user or a base station, it must first obtain a channel from one of the base stations that hears it if a channel is available, it is granted to the user. In the case that all the channels are busy, the new call is blocked. This kind of blocking is known as new call blocking and it refers to blocking of new calls. The procedure of moving from one cell to another, while a call is in progress, is called handoff. While performing hand off, the mobile user unit requests the base station of the cell that it moves into a new cell and will allocate it a channel. If no channel is available in the new cell, the handoff call is blocked. This kind of blocking is called handoff blocking and it refers to blocking of ongoing calls due to the mobility of the users.[19][20].

The Quality of Service (QoS) in cellular networks is mainly determined by these two quantities. The first determines the fraction of new calls that are blocked, while the second is closely related to the fraction of admitted calls that terminate prematurely due to dropout. A strong network backbone is needed to support high quality of service (QoS) without fully coordinated channel and network access is achievable. The wireless channel must be kept free from reaching the congestion point, since it will cause an overall channel quality to degrade and more loss rates to rise, leads to buffer drops and increased delays. Call admission control and network resource allocation are the key issues to determine the condition for accepting or rejecting a new call and handoff call based on the availability of sufficient network resources to guarantee the QoS parameters without affecting the existing calls. Call Admission Control basically deals with the acceptance and rejection of the incoming connection requests at the nearby Base Station Instead of blocking the calls immediately we proposed to use two buffers one for New calls and another for Handoff calls at each base station. The call requests are stored in the buffers until a specified time expires or the channel is made available to the call. Buffer Threshold deal with channel allocation. The Buffer Threshold depends on the rate at which the traffic enters the network. Giving higher priority to the handoff calls so as to keep the quality of service of handoff call .The Handoff calls will be provided with the channel first than the new calls. There may be a Link Failure In such situation we can provide another path for the communication to take place by applying a Restoration Mechanism for the survivability and connectivity of the channel for the communication. Here creating a new path from the node (Cell) after which the link is failure as virtual source and create a new path to the destination from this virtual source. This is called as Dynamic Path Restoration .Routing in Mobile Ad hoc Networks

The proposed model captures the differences between new call blocking and handoff blocking. Considering the movements of users along an arbitrary topology of cells. Under appropriate statistical assumptions, the system can be modeled as a multi-dimensional continuous-time Markov chain. However, there are two asymptotic regimes, one for very slow mobile users and another for very fast mobile users. The proposed protocol also deals with the issue of reliable multicast to reduce the maintenance overhead increase the path stability, reducing the congestion in mobile ad-hoc network and efficient use of bandwidth.

The rest of the paper is organized as follows. The problem statement is given in section 2 . The proposed model and algorithm to solve the problem is given in section 3 . The comparative results are discussed in section 4 Finally, conclusions and future work are discussed in Section 5 and 6 respectively.

\section{PROBLEM STATEMENT}

Cellular technology is designed to provide communication between two moving units, called as mobile units or between one mobile unit to one stationary unit called as land unit. The service provider must be able to locate and track a caller, assign a channel to the call, and transfer the channel to the call, and transfer channel from base station to another base station as the caller moves out of range. Each base station is controlled by a mobile switching centre .The mobile switching coordinate communication between all the base station and the central office. The central office is a computerized centre that is responsible for connecting calls, recording calls information When a call is started by a user first it goes to base station and assigned a channel by base station to this call if available if not it will be rejected. Here starts a problem to maintain and manage the path and bandwidth allocation in term of channel. In cellular networks, blocking occurs when a base station has no free channel to allocate to a mobile user. There are two kinds of blocking, the first is called new call blocking and refers to blocking of new calls, the second is called handoff blocking and refers to blocking of ongoing calls due to the mobility of the users. The explicit analytic expressions for the two kinds of blocking probabilities in two asymptotic regimes, one for very slow mobile users and another for very fast mobile users, and show the fundamental differences between these blocking probabilities. An approximation is introduced in order to capture the system behavior for moderate mobility.

The approximation is based on the idea of isolating a set of cells and having a simplifying assumption regarding the handoff traffic into this set of cells, while keeping the exact behavior of the traffic between cells in the set. It is shown that a group of three cells is enough to capture the difference between the blocking probabilities of handoff call attempts and new call attempts. [8] A new scheme for the call handoff problem in mobile cellular networks is introduced for Efficiently solving the handoff problem is important for guaranteeing quality of service to already admitted calls in the network. The scheme is based on a new approach called channel carrying when a mobile user moves from one cell to another, under certain mobility conditions, the user is allowed 
to carry its current channel information into the new cell. It proposes a new channel assignment scheme to ensure that this movement of channels will not lead to any extra co-channel interference or channel locking. The mobility of channels relies entirely on localized information, and no global coordination is required. Therefore, the scheme is simple and easy to implement it.[6]

The hybrid channel carrying scheme that allows to maximize performance under various constraints. The increasing demand for mobile services has generated worldwide interest in wireless communication networks. Coupled with this interest comes the consumer expectation that the wireless systems provide comparable quality of service to their wired counterparts. Studies have shown that one of the most important user concerns is that service is not be cut off during an ongoing call. It addresses this concern by proposing a new scheme to achieve efficient call handoffs in wireless cellular networks. The use of cellular systems has been a very popular means of enhancing the capacity of wireless communication networks. In such a system, the service area is divided into cells, and channels are reused among those cells. Here, a channel is referred to as the unit of wireless spectrum needed to serve a single user. For example, in a time-division multiplex /frequency-division multiplex access system, a time-slot, or carrier frequency can be considered as a channel. Channels that are used in one cell cannot be used in other cells that are closer than the minimum reuse distance. Handoff occurs when a mobile subscriber moves from one cell to another. A handoff call may be blocked if there is no free channel in the new cell. Since blocking a handoff call is less desirable than blocking a new call, septic schemes have been developed to prioritize handoff calls. Two prioritization schemes have been commonly studied in the literature are as follows.

1) Channel Reservation Scheme: A number of channels are reserved solely for the use of handoff, allowing both handoff and new calls to compete for the remaining channels Specifically, in each cell a threshold is set, and if the number of channels currently being used in the cell is below that threshold, both new and handoff calls are accepted. However, if the number of channels being used exceeds this threshold, an incoming new call is blocked and only handoff calls are admitted.

2) Queuing Scheme: A handoff requests are queued and may later be admitted into the network in case a channel frees up.

The above two schemes can also be integrated together to improve the handoff blocking probability and the overall channel utilization. The scheme propose in this paper is also readily integrated with the queuing schemes. The method for treating the handoff problem stems from the following simple idea. A user requesting a handoff always occupies a channel in its current cell. Therefore, if the channel could be carried into the new cell, the handoff request would not be blocked. When we say a channel is "carried" into a new cell, we mean that the mobile user continues to use this channel, but now communicates with the new base-station as the new cell.
Whenever a link failure occurs It deals with backup path restoration to continue the communication in the fixed part of the network. This scheme tries to automatically maintain the link for better QoS. The path restoration can be done in to following two ways Static restoration and Dynamic restoration.

The Static restoration deals with allocation of the backup path at the time of the allocation of the channel to the call. If the link currently being used fails the backup path is utilized for communication to continue. The major problem with static scheme is that each communication uses an extra channels and this lead to poor throughput of the network.

To over come this wastage of the channel the Dynamic restoration is used .This deals with dynamic restoration of the link if the link failure occurs. Thus it avoid the double channel allocation at the time of the channel allocation for the call.

\section{A. CALL ADMISSION CONTROL POLICY}

There are several research work has been done based on call admission control and buffer management for different traffic classes in wireless networks. There are following policy that takes only local information in the admission decision process, and therefore will have a high call dropping probability. To reduce the call dropping probability, few CAC algorithms are considered having neighboring cells information However, these Algorithms only support users with fixed bandwidth requirements. Employed the concept of prioritization of handoff calls over new calls by using buffering technique since it is desirable to complete an ongoing call rather than accepting a new one .Developed a class-based admission policy that satisfies the QoS requirements for each traffic class by allocating adequate resources to each type. The Call Admission Control schemes in [21] and single buffer management were designed for the purpose of congestion control in the mobile network, little or no work has been carried out on their combined effects as a resource manager.[22 ] Hence, it does not explore all the possible benefits a well designed CAC scheme has to offer. Therefore, it would be worthwhile and beneficial to explore how different CAC schemes manage to improve the network utilization, and how well they can be combined with buffer. This could permit direct comparison and allow for the determination of the best scheme as well as presenting how buffer could provide even greater results.

\section{B. HANDOFF CALL CONTROL POLICY}

The Call Admission Control techniques Proposed uses only single buffer for handoff calls but for new calls there is no buffer [ 23][24][25]. This means that if no channel is available for the new call request it will be rejected without waiting for the channel to be allocated. In accordance the handoff call would be allocate channel if the channel is available otherwise it will be buffered. The channel to these handoff calls will be allocate at any time only if the following condition occurs.

- User completes the call

- The user moves to the another cell before the call is completed 
It required to check all the time for all the calls (both new and handoff calls) that total channel available to is not less than the channel requirement of the incoming call requests.

The proposed scheme consider about new call blocking problem when a new call is introduced by base station it will be check for availability of channel if it is there then allocate it otherwise put into a buffer. This implementation provide a quality of service guarantee to both new and handoff calls and at the same time improve the performance of the network. If a link failure occurs in the network path, while a call is in progress, then the call gets terminated. This leads to low Quality of Service Restoration Mechanism is proposed for the survivability and connectivity of the channel for the communication. Here creating a new path from the node (Cell) after which the link is failure as virtual source and create a new path to the destination from this virtual source. This is called as Dynamic Path Restoration .Routing in Mobile Ad hoc Networks[26][27][28]

\section{3 PROPOSED MODEL OF HETEROGENEOUS NETWORK}

The heterogeneous mobile Network has with available bandwidth $B$ at each base station and number of mobile nodes be $\mathrm{n}$ and distance between heterogeneous Base station nodes is D and load at each Base station node be L.

The following figure shows the wireless network of five Heterogeneous Base Station nodes

$\mathrm{B}=$ Total Available nodes Bandwidth

$\mathrm{n}_{\mathrm{i}}=$ Nodes Name (Base Station)

$\mathrm{Q}_{\mathrm{i}}=$ Length of queue at Base Station node $\mathrm{n}_{\mathrm{i}}$

$\mathrm{M}_{\mathrm{i}}=$ Total number of Mobile users at node $\mathrm{n}_{\mathrm{i}}$ (Base Station).

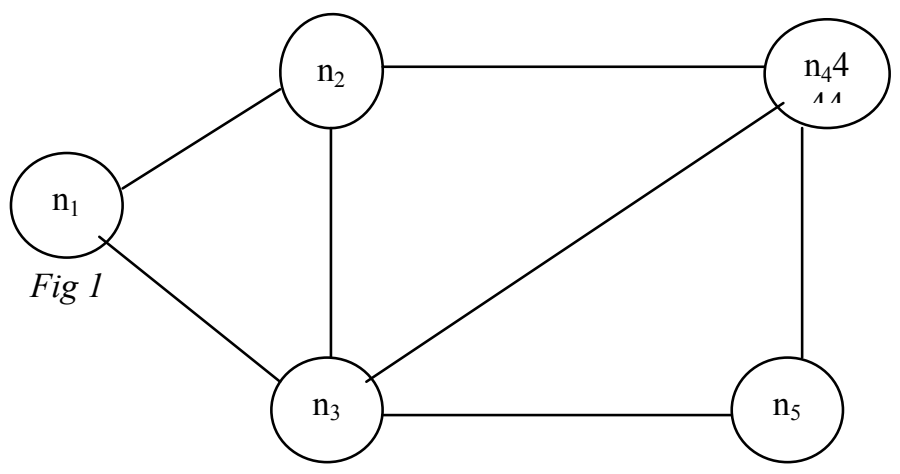

So in order to select path from $\mathrm{n}_{1}$ to $\mathrm{n}_{5}$

1. $P_{1}: n_{1}-n_{2}-n_{4}-n_{5}$, or

2. $\mathrm{P}_{2}: \mathrm{n}_{1}-\mathrm{n}_{3}-\mathrm{n}_{5}$, or

3. $\mathrm{P}_{3}: \mathrm{n}_{1}-\mathrm{n}_{2-} \mathrm{n}_{3}-\mathrm{n}_{4}-\mathrm{n}_{5}$, or

4. $\mathrm{P}_{4}: \mathrm{n}_{1}-\mathrm{n}_{2}-\mathrm{n}_{3}-\mathrm{n}_{5}$, or

5. $\mathrm{P}_{5}: \mathrm{n}_{1}-\mathrm{n}_{3}-\mathrm{n}_{4}-\mathrm{n}_{5}$, or

6. $\mathrm{P}_{6}: \mathrm{n}_{1}-\mathrm{n}_{2}-\mathrm{n}_{4}-\mathrm{n}_{3}-\mathrm{n}_{5}$

\section{A. BASE STATION PATH SELECTION} CHARACTERISTIC

1. Distance of selected path is minimum or optimum.

2. Load in selected path is minimum or optimum and load at intermediate node is less than threshold of B.

3. Queue length at intermediate nodes of the path is minimum or optimal.

Distance is based on the number of hop counts .Queue Length is known to all the nodes, while transferring the queue length, the maximum of all queue length at intermediate nodes in path is stored only. As a node can transfer only one packet at time, thus the queue length can be used to estimate the available bandwidth, as we are not considering multiplexing of data at the nodes. Thus, the position of paths in bandwidth list will be similar to the position of path in queue length list.

\section{B. CONTROL PACKET DETAILS}

The control packet has the following sections.

\section{1) CONSTRUCTION OF ROUTING TABLE}

Whenever a mobile node enters in a wireless network it would broadcast a notification packet with fields as shown in fig. -2

\begin{tabular}{|l|l|l|l|}
\hline Node No. & Distance & $\begin{array}{l}\text { Queue } \\
\text { length }\end{array}$ & $\begin{array}{l}\text { Flag } \\
(00)\end{array}$ \\
\hline
\end{tabular}

Fig. -2 Notification packet

Initially the distance field value is initialized to 1 and queue length is initialized to 0 and node number is calculated from the IP address and subnet mask. Arithmetic to calculate the node number is to apply AND operation on complement of subnet mask and IP address. Flag field is a 2-bit field and its set to 00 for notification packet. The receiving node, would match node no. of the received packet from their table, if it don't have this node no. registered in its table, it would add a row. An example of table construction is shown in Fig. - 3

\section{2) CONSTRUCTION OF PATH}

Now when a nodes get a packet to transmit to some other node then it calculate the node number of the destination node, if it has entry of this node in its routing table then it would simply send packet to it otherwise it would broadcast the route request(RREQ) packet(Fig. - 4) with unique sequence no., its node no. as the sender and route source node no. the flag is set as 01 for RREQ. The node receiving RREQ would then check its table for destination node, if it has entry then it sends the route reply packet (RREP) (Fig. - 5) packet with its sequence no. as that of RREQ packet, it's node number in the path field, distance increased by one from the value in its routing table in the distance field, it's queue length in the queue length field 
and flag as 10 to the sender node number of RREQ packet, and then multicast the RREQ packet with changed sequence no and its node no. as sender node no. to all the nodes in its routing table except of sender node no. and destination node no and save this information of original sequence no., modified sequence no. sender node no. and route source node no. in its memory. Even if the node receiving RREQ does not have the entry of destination node in its routing table then also it would multicast it to other node, in its table, except for sender node, route source node number and destination node no in the same manner as described before.

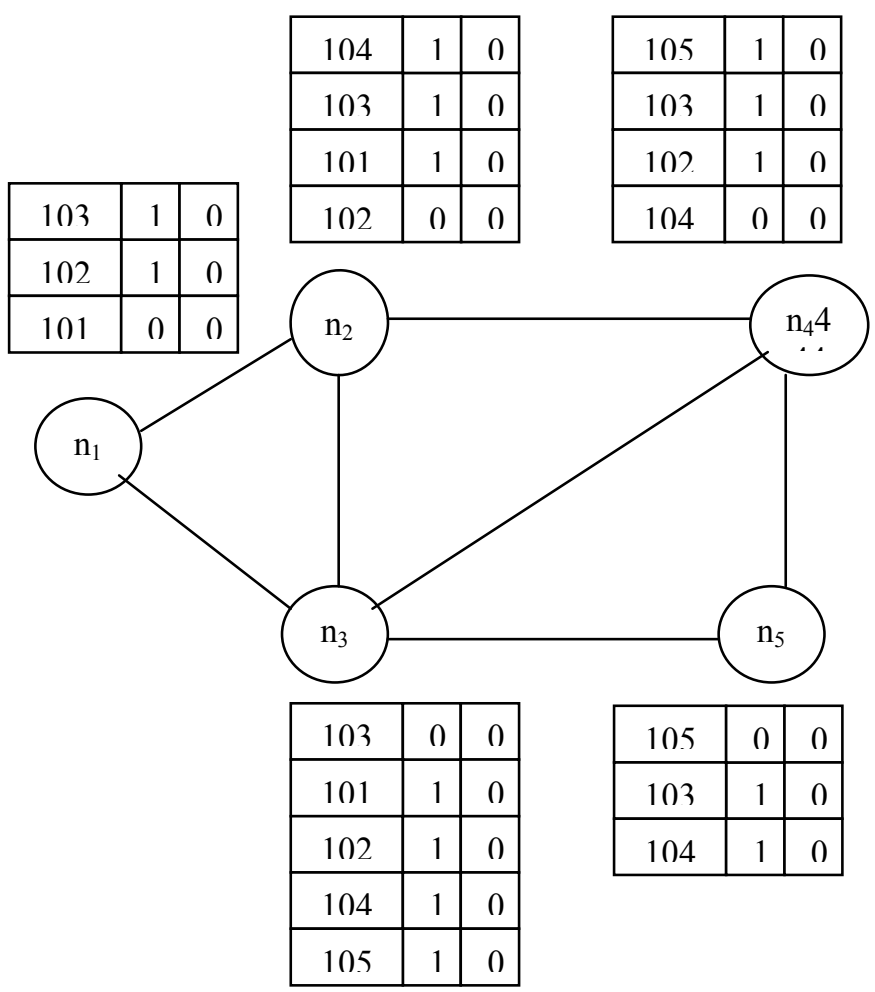

Fig. - 3 Construction of Routing Table

The node receiving route reply packet checks to see if it itself is route source node number. If it is not the route source node no. then it would match the sequence no. and route source node no. in its memory to retrieve the original sequence no. and sender node no. and it sends the route reply packet to the sender node no. with sequence no. as retrieved sequence no. and append its own node no. to the path data field and queue field value is set either to value of queue length field of received RREP packet or the its queue length value from the routing table depending upon whichever is maximum

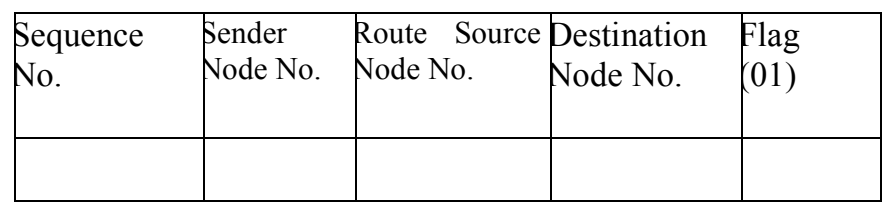

Fig. - 4 Route Request Packet (RREQ)

\begin{tabular}{|l|l|l|l|l|l|l|}
\hline $\begin{array}{l}\text { Sequ } \\
\text { ence } \\
\text { No. }\end{array}$ & $\begin{array}{l}\text { Path } \\
\text { data }\end{array}$ & $\begin{array}{l}\text { Route } \\
\text { Source } \\
\text { Node } \\
\text { No. }\end{array}$ & $\begin{array}{l}\text { Destinati } \\
\text { on Node } \\
\text { No. }\end{array}$ & Distance & $\begin{array}{l}\text { Queue } \\
\text { Length }\end{array}$ & $\begin{array}{l}\text { Flag } \\
(10)\end{array}$ \\
\hline & & & & & & \\
\hline
\end{tabular}

Fig. - 5 Route Reply Packet (RREP)

\begin{tabular}{|l|l|l|l|l|}
\hline Path data & $\begin{array}{l}\text { Destination } \\
\text { Node No. }\end{array}$ & $\begin{array}{l}\text { Route } \\
\text { Source } \\
\text { Node No. }\end{array}$ & Data & $\begin{array}{l}\text { Flag } \\
(11)\end{array}$ \\
\hline
\end{tabular}

Fig. - 6 Message Packet

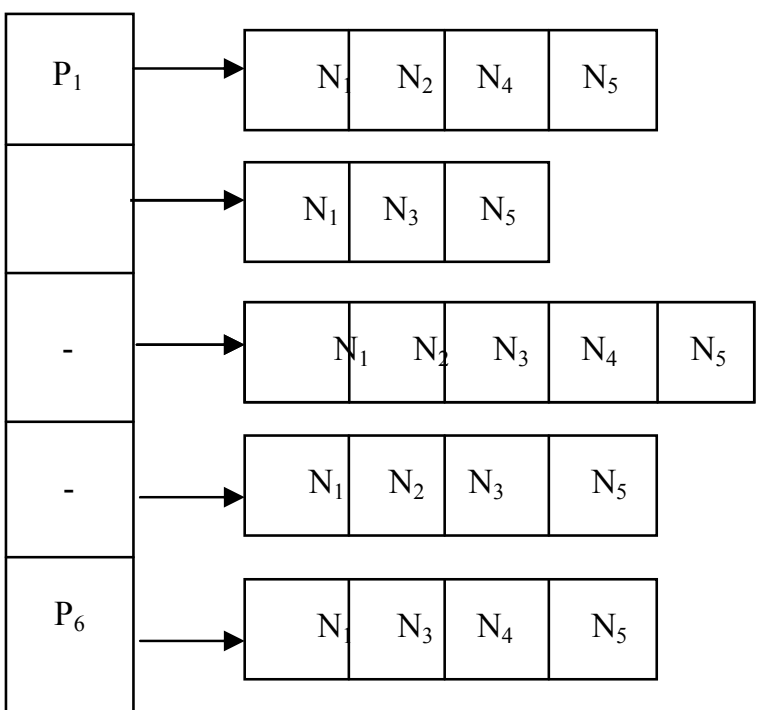

Fig. - 7 Path list at the Route Source Node

The route source node on receiving any of the, RREP packet will save it in its path list as shown in Fig. -7

3) ALGORITHM FOR MAINTENANCE OF ROUTES

Input : Routing Table: RTable [] [], MessagePacket :M[],

Destination Node No. : D_node , Boolean variable Flag $=0$

1. Start

2. Len=Length[M]

3. If $((\mathrm{M}[$ Len -2$]==1)$ AND $(\mathrm{M}[$ Len -1$]==1)$ )$/ *$ Message packet received $* /$

4. For $\mathrm{I}=0$ to Length [RTable] 
5.

6.

7.

If (RTable [i] [0] = = D_node )

Transmit $\mathrm{M}$ to $\mathrm{D} \_$node

Flag $=1$

8. Break

9. $\quad$ End If

10. End For

11. If (! Flag )

12. Broadcast RREQ packet with field values as Seq (Sequence NO. $)=$ System generated no.

S_No.( Sender node_no.) $=$ self node no.

Rs No. ( Route Source Node no. ) = Self Node

D_no. (Destination Node No. ) = D_node

13. End If

$$
\mathrm{F}(\mathrm{Flag})=01
$$

14. If $((\mathrm{M}[$ Len -2$]==0)$ AND $(\mathrm{M}[$ Len -1$]==1$ )) )/*Route request packet received*/

15. For $\mathrm{I}=0$ to Length [RTable]

16. If (RTable [i] [0] = = D_node )

17. Send RREP to S_No. with Field values

Seq $($ Sequence No $)=$ RREQ.Seq

$\operatorname{Pd}($ Path Data $)=$ stack implementation ( with self node no on top )

Rs No. ( Route Source Node no. ) = RREQ.Rs No.

D_no. (Destination Node No. $)=$ RREQ . DNo.

$\mathrm{D}($ Distance $)=$ RTable [i] [1] +1

Q_Len (Queue Length) = RTable [i] [2] $\mathrm{F}($ Flag $)=10$

18. $\quad$ Flag $=1$

19. Break

20. End If

21. End For

22. If ( ! Flag )

23. Multicast RREQ packet to all except for sender node no, route source node no and destination node no with field values

Seq (Sequence NO. ) = System generated no. S_No.(Sender node_no.) $=$ self node no.

Rs No. ( Route Source Node no. ) = Self Node D_no. (Destination Node No. ) = D_node

$$
\mathrm{F}(\text { Flag })=01
$$

24. Make an entry in system database with field values

New_Seq $=$ Seq in step 23

Old_Seq $=$ RREQ.Seq

RS_No. = RREQ.RS_No.

D_No. $=$ RREQ.D $>$ No.

Sender $=$ RREQ.SNo.

25. End If

26. If $((\mathrm{M}[$ Len -2$]==1)$ AND $(\mathrm{M}[$ Len -1$]==0$

)) /*Route reply packet received*/
27. If ( RREP.RS_No. $==$ Node_No.)

28. Add Path data of RREP to the path Linked List at the node.

29. Else

30. Insert its node no. in path data of RREP

31. If ( RREP.Q_Len $<$ RTable[0][2])

32. RREP.Q_Len=RTable[0][2]

33. End If

34. Retrieve sender node no. and Sequence number from database by RREP.Seq, RREP.S_no.

35. RREP.Seq=Sequence no. of step 33

36. Send RREP to sender node of sep 33

37. End If

38. End If

39. Stop.

4) ALGORITHM FOR SELECTION OF PATH

Consider all the following paths condition

i) Arrange all the possible paths in ascending order of queue length, load and distance, considering only paths which has load lower than threshold.

ii) Take the sum of position of the path in the three lists and finally select the path with lowest sum.

iii) In case if minimum sum of position in the three lists calculated in step (ii) is more than once then the following preference order is used to break the tie for selecting an optimal path.

\section{Queue Length $>$ Load $>$ Distance of path}

The queue length (Bandwidth Concept) of each node in the fig-1 is as follows

$\mathrm{Q}_{1}=10, \mathrm{Q}_{2}=12, \mathrm{Q}_{3}=15, \mathrm{Q}_{4}=9, \mathrm{Q}_{5}=5$

Thus the queue length and distance of paths are shown in table-1 as details of Paths.

\begin{tabular}{|c|c|c|}
\hline Distance & Path & $\begin{array}{c}\text { Queue } \\
\text { Length }\end{array}$ \\
\hline 3 & $\mathrm{P}_{1}: \mathrm{n}_{1}-\mathrm{n}_{2}-\mathrm{n}_{4}-\mathrm{n}_{5}$ & 10 \\
\hline 2 & $\mathrm{P}_{2}: \mathrm{n}_{1}-\mathrm{n}_{3}-\mathrm{n}_{5}$ & 15 \\
\hline 4 & $\mathrm{P}_{3}: \mathrm{n}_{1}-\mathrm{n}_{2}-\mathrm{n}_{3}-\mathrm{n}_{4-}$ & 15 \\
\hline 3 & $\mathrm{P}_{4}: \mathrm{n}_{1}-\mathrm{n}_{2}-\mathrm{n}_{3}-\mathrm{n}_{5}$ & 15 \\
\hline 3 & $\mathrm{P} 5: \mathrm{n} 1-\mathrm{n} 3-\mathrm{n} 4-\mathrm{n} 5$ & 15 \\
\hline 4 & $\mathrm{P} 6: \mathrm{n} 1-\mathrm{n} 2-\mathrm{n} 4-\mathrm{n} 3-\mathrm{n} 5$ & 15 \\
\hline
\end{tabular}

Table 1

Arranging the paths in ascending order with respect to Distance, load and queue length as follows in the table with their position 


\begin{tabular}{|c|c|c|c|}
\hline Position & Distance & Load & $\begin{array}{c}\text { Queue } \\
\text { Length }\end{array}$ \\
\hline 1 & P2 & P1 & P1 \\
\hline 2 & P1 & P2 & P2 \\
\hline 3 & P4 & P3 & P3 \\
\hline 4 & P3 & P4 & P4 \\
\hline 5 & P5 & P5 & P5 \\
\hline 6 & P6 & P6 & P6 \\
\hline \multicolumn{4}{|c}{ Table 2 } \\
\hline
\end{tabular}

The sum of position of path in the three lists (distance, load and queue length)

For $\mathrm{p}_{1}:(2+1+1)=4$

For $\mathrm{p}_{2}:(1+2+2)=5$

For $\mathrm{p}_{3}:(4+3+3)=10$

For $\mathrm{p}_{4}:(3+4+4)=11$

For $\mathrm{p}_{5}:(5+5+5)=15$

For $\mathrm{p}_{6}:(6+6+6)=18$

From above calculation it is clear that the sum of position of path $\mathrm{P}_{1}$ in the three lists is minimum hence path $\mathrm{P}_{1}$ is selected.

\section{DESIGN SPACE AND PROPOSED ALGORITHM FOR HANDOFF/NEW CALL}

The proposed scheme considers a multiple class of calls (multimedia system) that differ in their QoS requirements and traffic parameters, allowing for call transitions among classes. The model is structured over users moving along an arbitrary topology of cells. Each cell has the same number of channels, due to the fact that wireless network resources are limited, which give service to classes of call request that is assumed to be generated according to a Poisson distribution with an exponential call holding time, during call establishment, a call is assumed to declare its priority. The call processing entities of the system are the processing elements of the Base station, different Base Station Controller are able to identify the call type at any moment. The available resources are maximum number of channels in a cell and the buffer size that is used to queue handoff call in case no channel is available. The buffer size could be adjusted depending on the input traffic rate. The proposed model is as follows. It is clear from the figure -8 that there are two buffers one for the handoff calls and other for the new calls. If channel is not available then the calls will be buffered into their corresponding buffers and as soon as the channel is available it is allocated to the calls .The calls buffered in to the buffers are allowed to wait for a specified period of time after which it will be time the channel is made available to the calls then it will get the channel allocated.[29]

We use the proposed algorithm to get shortest path for communication to occur with minimum delay. The following system performance parameters are used for congestion control.

1. New call blocking probability,
2. Handoff blocking probability,

3. Call holding time, and

4. Buffer size.

To get effective deployment of congestion controlled a relationship among these parameters is established. A threshold value is used to deal with channel allocation to the new and handoff calls. This threshold depends on the traffic in the network

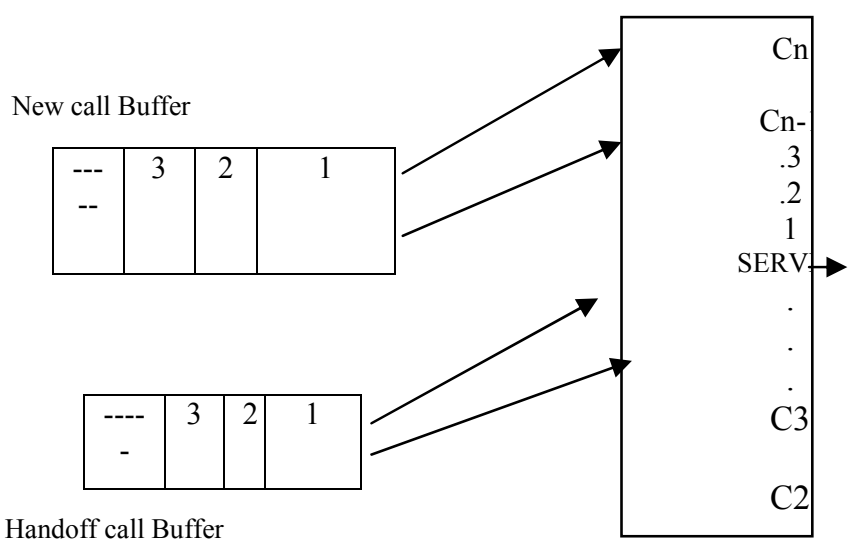

Fig-8

For example generally a customer waits in a queue with other customers awaiting service, but it is possible for the seller to provide higher priority to certain, presumably higher spending customers. Computational resources could be done in the same manner. Handoff call would be buffered if no free channel is available rather than rejecting them. This would be allocated to channel any time either user completes the call / the user moves to another cell before the call is completed. We are using a new call buffer and handoff buffer for providing a better QoS and for better channel utilization. The new call is allowed to wait accordingly its dwell time .As soon as the dwell time expires the call is rejected. However, the handoff will be allocated channel first depends on the priority attached to it. The policy makes us determine whether delay sensitive handoff should be buffered, or allowed to be initiated again based on its dwelling time. The algorithm is as follows

\section{ALGORITHM FOR NEW CALL / HANDOFF CALL}

If (incoming request is new call or handoff call) then

If (there is a free channel) then

Allocate the free channel

/* Channel allocation to all the calls either it is new call or handoff call, if channel is available*/

Else

If (handoff call or newcall) then

If ( handoffcall ) then

put in handoff_buffer

/*buffering the handoff calls*/ 
Endif

If(newcall) then

put in newcall_buffer

/*buffering the new calls*/

Endif

Endif

If (there is free channel again and newcall_priority<threshold) then

allocate the free channel to

handoff call

/*channel allocation to handoff calls*/

Increment newcall_priority

Else if(there is free channel and newcall_priority $>=$ threshold)

then

Allocate the free channel to new call

$/ *$ channel allocation to new calls*/

Else

Ignore request

Endif

Endif

Ignore request

Endif

End.

\section{DYNAMIC LINK RESTORATION TECHNIQUE}

Dealing with link failure in the cell of the network we proposed the Dynamic Link Restoration technique. This technique is restoring the link failure dynamically during the call is in progress. Link restoration establishes a new route only between the end nodes of the failed link while still using the rest of the links in the old path. The Dynamic link restoration technique dynamically restores the failed link and provides the transparent protection to the end nodes of the failed link. In this way the link is going to be dynamically maintained without the knowledge of the users that is all this process is done transparent to the users. While link restoration there might be the situation that no channel is available in the neighborhood of the node after which the link has failed or the node before which the link has failed. In such situation the call will get terminated. But the probability of occurrence such condition is very less. [30]

\section{SIMULATION RESULTS}

In our simulation, a flat heterogeneous network is assumed as clusters. For unicast, before a mobile user node sends a unicast packet, it sets RTS (Request-to- Send) flags of its neighbors and the intended receiver sets CTS (Clear-to-Send) flags of its neighbors. Nodes whose RTS or CTS flag is set cannot transmit data, except the sender. When the sender finishes sending the data, RTS/CTS flags are cleared by the nodes which originally set those flags. Similar scheme is used in multicasting .The node wants to send a multicast packet sets RTS flags of its neighbors, and each intended receiver sets CTS flags of its neighbors. The broadcast uses flooding, technique and only RTS flags are set by the sending node, and CTS flags are not set by any node. Therefore, in broadcast, collision may occur. However, collisions are ignored in our simulation. The simulated network area is a $\mathrm{N} \mathrm{x} \mathrm{N} \mathrm{meter}$ square, and $\mathrm{M}$ mobile nodes are roaming randomly in all directions at a predefined speed in this area. Each Base station node has two finite buffers, and new call and handoff call packets are lost when buffer overflow occurs. New call and Handoff call control packets have higher priority over data packets in our simulations. Propagation delay is assumed to be negligible, and it is assumed that packets always arrive without any bit error. Extensive simulation results obtained by varying several network parameters and workload configuration. The values of the network parameters used in simulations are those specified in the IEEE 802.11. In this scenario we evaluate the performance improvement in terms of throughput ie. call completed in a densely populated network. Specifically, we consider a network of 5 to 40 Base Stations with 20 to 80 mobile user at each base station nodes with an increasing number of neighbors from 5 to 40 Base Station. Each node has a traffic flow with infinite demands towards one of its neighbors. In Fig. 9 to Fig. 16. We show the some of throughput of all traffic flows, with available Channels Bandwidth.

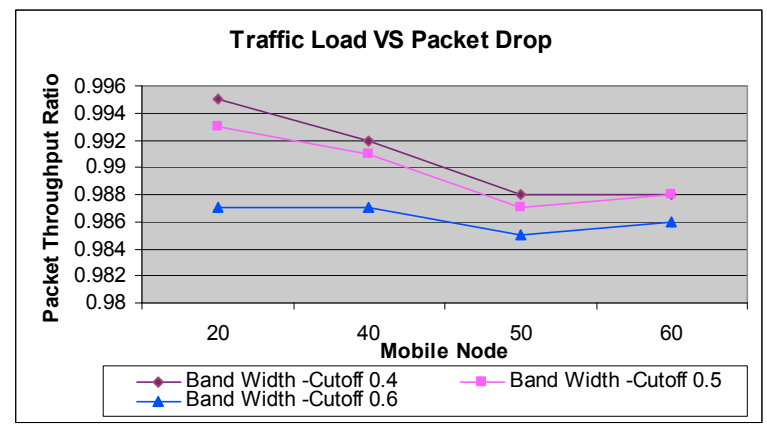

Fig-9



Fig-10 


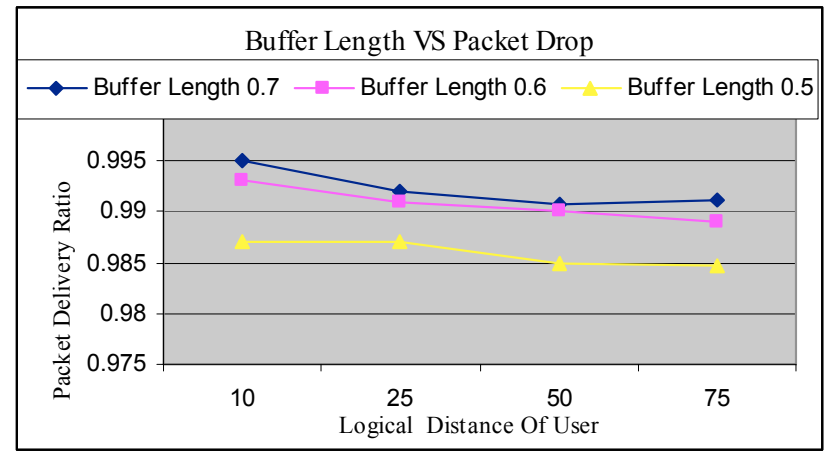

Fig-11

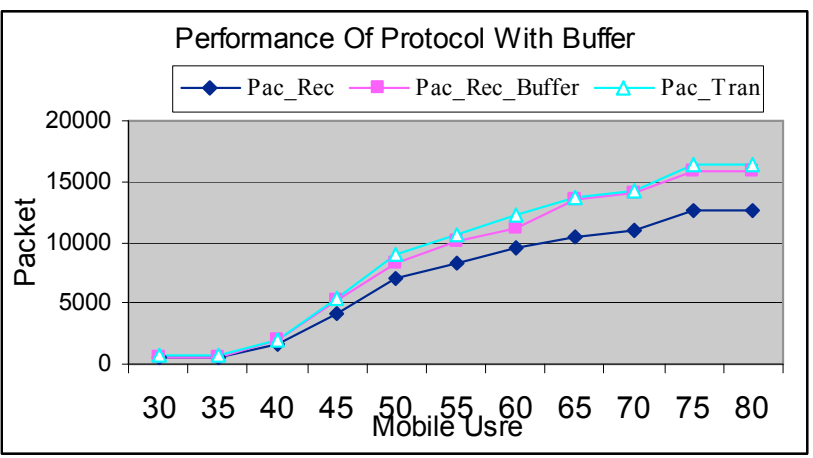

Fig-12

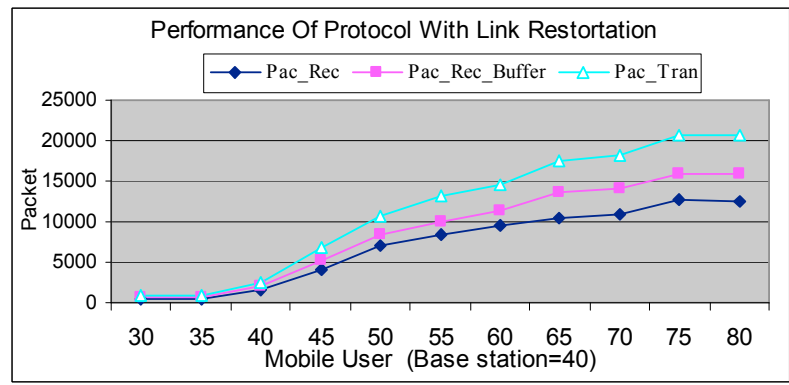

Fig-13

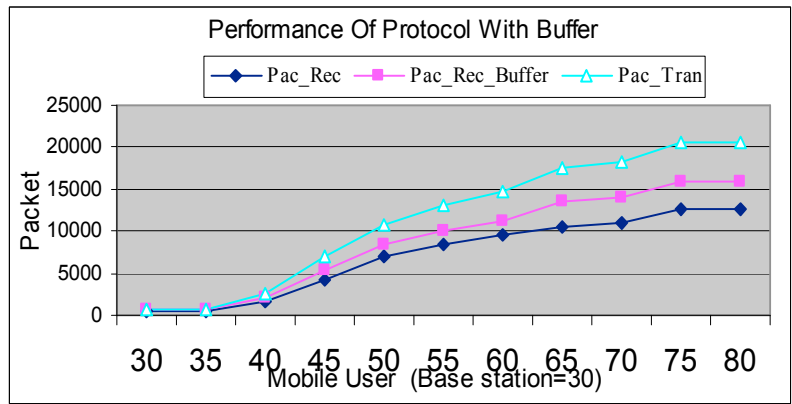

Fig-14



Fig-15

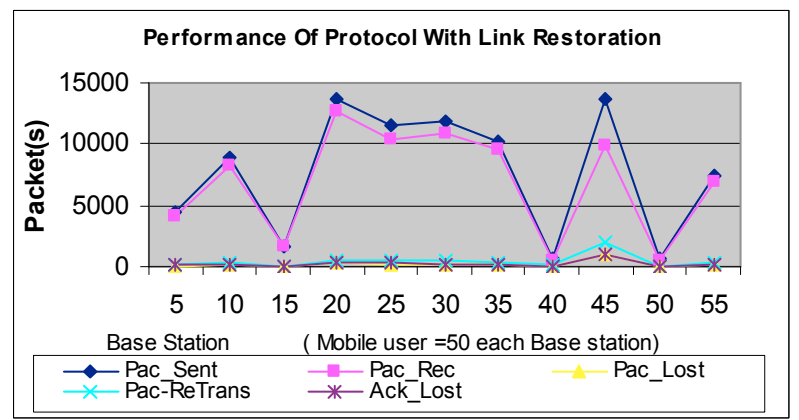

Fig-16

\section{CONCLUSION}

The proposed Protocol for wireless mobile heterogeneous networks for measuring the performance of call admission control for new call blocking, handoff call blocking, call holding time and buffer size are improved. It mostly selects the optimal path for transmission of packets from source to destination in heterogeneous networks. The Protocol reduce handoff call blocking probability and new call blocking probabilities by using two buffers, one for new call and another buffer is use for handoff calls if there is no channel available instead of dropping it store in the buffer and when the channel is free it allocate for communication The effect of the dynamic threshold of buffer size of new call buffer and handoff call buffer and dynamic link restoration technique is improved the performance of the heterogeneous networks.

\section{FUTURE SCOPE}

In future there can be further evaluation of our scheme by using more realistic mobility of nodes in the simulation. We believe the advantage of providing traffic information will be significant in those environments.

\section{REFERENCES}

[1] D. B. Johnson and D. A. Maltz. Dynamic source routing in ad-hoc wireless networks. Mobile Computing, chapter 5, Kluwer Academic Publishers, 1996.

[2] C-K Toh. Wireless ATM and Ad-Hoc Networks: Protocols and Architectures. Kluwer Academic Publishers, 1997.

[3] C. E. Perkins and P. Bhagwat. Highly Dynamic Destination Sequenced Distance Vector Routing (DSDV) for Mobile Computers, SIGCOMM Conf. Proc, 1994.

[4] S. Corson and A. Emphremides. A Distributed Routing Algorithm for Mobile Wireless Networks, ACM/Baltzer Wireless Networks J., vol. 1, no.1, 1995. 
[5] S. Murthy and J. J. Garcia-Luna-Aceves. A Routing Protocol for Packet Radio Networks, MOBICOM, 1995.

[6] R. Dube et. Al. Signal Stability-Based Adaptive Routing (SSA) for Ad Hoc Mobile Network, IEEE Personal Communications, 1997.

[7] Z. J. Hass. A New Routing Protocol for the Reconfigurable Wireless Network, ICUPC, 1997.

[8] S. E. Deering and D. R. Cheriton. Multicast Routing in Datagram Internetworks and Extended LANs. ACM Transaction on Computer Systems, May 1990.

[9] C-C. Chiang and M. Gerla. On-Demand Multicast in Mobile Wireless Networks. Proceedings of IEEE ICNP '98, 1998.

[10] C-C. Chiang and M. Gerla. Routing and Multicast in Multihop, Mobile Wireless Networks. Proceedings of ICUPC '97, 1997.

[11] C-C. Chiang, M. Gerla and L. Zhang. Shared Tree Wireless Network Multicast. Proceedings of IEEE 6th International Conference on Computer Communications and Networks (ICCCN'97), 1997.

[12] C. E. Perkins. Ad-hoc On Demand Distance Vector (AODV) routing. Internet-Draft, draft-ietf-manet-aodv- 00.txt, November 1997.

[13] David B. Johnson and David A. Maltz. Dynamic source routing in ad hoc wireless networks. Technical report, Carnegie Mellon Unity, 1996.

[14] D. Waitzman, C. Partridge, and S. Deering, "Distance Vector Multicast Routing Protocol (DVMRP)", RFC 1075, Nov. 1988

[15] B. Quinn, and K. Almeroth, "IP Multicast Applications: Challenges and Solutions", RFC 3170, Sep. 2001

[16] C. E. Perkins and P. Bhagwat. Highly dynamic Destination- Sequenced Distance-Vector routing (DSDV) for mobile computers. Proceedings of the SIGCOMM '94 Conference on Communications Architecture, Protocols and Applications, page 234-244, August 1994.

[17] T. Ballardie, P. Francis, and J. Crowcroft. Core Based Tree (CBT) an architecture for scalable interdomain multicast routing. Proceeding of ACM SIGCOM, 1993.

[18] A. Ballardie, "Core Based Trees (CBT version 2) Multicast Routing Protocol Specification”, RFC 2186, Sep. 1997.

[19] C. PERKINS, E.ROYER AND S. DAS Ad hoc On-demand Distance Vector (AODV) Routing, RFC

[20] E. M. Royer and C. E. Perkins. Multicast Ad hoc On- Demand Distance Vector (MAODV) Routing . draftietf. manet-maodv-00.txt, July 2000.

[21] T. Ozaki, J. B. Kim, and T. Suda, "Bandwidth-Efficient Multicast Routing for Multihop, Ad-Hoc Wireless Networks", in IEEE INFOCOM, 2001.

[22] T. Sirin, "A Measurement-Based Prioritization Scheme for Handovers in Mobile Cellular Networks", IEEE JSAC

[23] "Development of congestion control scheme for wireless Mobile network" by Oyebisi T.O and Ojesanmi O.A. in the "Journal of Theoretical and Applied Information Technology", 2005- 2008, www.jatit.org

[24] T. E. Mahmoud, M. E. Khaled and M.A. Mohamed, "Performance Analysis and Estimation of call Admission Control parameters in wireless Integrated Voice and data networks", IEEE Communication Magzine, vol. 3, No. 11,1991, pp. 42-46

[25] "Routing with Congestion Awareness and Adaptively in Mobile Ad hoc Networks" by Duce A. Tran and Harish Raghavendra

[26] "A novel Approach of Backup path restoration for survivable HighSpeed Networks" by Chi-Chun Lo,Bin-Wen Chuang, National ChiaoTung University, IEEE Communication Magzine, March 2003

[27] "A Novel Handoff Scheme for Mobile Cellular Network" - Junyi Li, Ness B. Shroff and Edwin K. P. Chong. IEEE/ACM TRANSACTIONS ON NETWORKING, VOL.7, NO. 1, FEBRUARY 1999.

[28] "Admission Control for Multi-services Traffic in Hierarchical MobileIPv6 Networks using Fuzzy Inference System” Shun-Fang Yang ,Bor Jiunn Hwang.- The International conference on Mobile Technology, Application \&System 2008(Mobility Conference), 10-12 September, 2008,Ilan, Tiawaan.2008 ACM 978-1-60558-089-0

[29] "New Call Blocking versus Handoff Blocking in Cellular Networks "Moshe Sidi ,and David Starobinski. Wireless Networks 3 (1997) 1527. J.C. Baltzer AG, Science Publishers.
[30] S. Choi and K.G. Shin, "Predictive and Adaptive Bandwidth Reservation for Handoffs in QoS- Censitive Cellular Networks", In ACM SIGCOMM'98 Proceedings, 1998, pp. 155-166. 